\title{
Central venous catheterisation in very low birthweight infants
}

\author{
H MACTIER, L G ALROOMI, D G YOUNG, AND P A M RAINE \\ Glasgow Royal Maternity Hospital and Royal Hospital for Sick Children, Glasgow
}

SUMMARY Forty two premature babies (mean birth weight $980 \mathrm{~g}$, mean gestation 27.6 weeks) had central venous lines inserted at a mean age of 10 days through the internal jugular vein because of poor peripheral venous access and for purposes of parenteral feeding and minimal handling. Eight babies died from complications of prematurity and four from septicaemia with a central line in situ, but the other 30 babies had lines in place for a mean of 20 days. A mean weight gain of $17 \cdot 5 \mathrm{~g} / \mathrm{kg} /$ day was recorded. Eight babies showed signs of infection at a mean of 22 days after insertion of the line. The other complications were thrombosis related to the catheter (three cases), embolisation (two), and hydrocephalus related to superior vena caval thrombosis (one). The policy of management is outlined, and the risks and benefits of the technique are analysed.

The increasingly prolonged survival of extremely premature, very low birthweight infants with immature gastrointestinal tracts and high requirements for blood products, parenteral nutrition, and antibiotics means that venous access is often a major problem in the busy neonatal unit. The case records of 42 infants with a birth weight under $1500 \mathrm{~g}$ in whom central venous catheterisation was performed were reviewed to investigate whether this is an acceptable method of providing reliable venous access for these very high risk babies.

\section{Patients}

Between December 1979 and February 1985, 42 central venous long lines were inserted in 42 neonates ( 21 boys and 21 girls) with a birth weight under $1500 \mathrm{~g}$ (range 700-1420 g). Mean (SD) birth weight was 980 (178) $\mathrm{g}$ and mean (SD) weight at insertion of the line was $995(221) \mathrm{g}$. The gestational age was estimated from maternal obstetric records and clinical examination of the baby ${ }^{1}$ and ranged from 25 to 32 weeks (mean (SD) $27.6(1 \cdot 7)$ ). The mean (SD) age at insertion of the line was $10 \cdot 7$ $(11.8)$ days; 22 lines $(52 \%)$ were inserted in the first week of life. Thirty one babies $(74 \%)$ were receiving mechanical ventilation when the line was inserted. The indications for insertion of a central venous line were: (a) an anticipated requirement for prolonged intravenous alimentation after removal of an um- bilical arterial or venous catheter; (b) to minimise handling of very sick, low birthweight infants; (c) poor peripheral venous access, making insertion of fine bore silicon long lines (Vygon code number 2184.06) impossible.

\section{Technique}

The lines were inserted with the babies in a supine, neck extended position in the incubator in the neonatal intensive care unit. Aseptic techniques, preparation of skin with povidone-iodine, and local anaesthesia with $1 \%$ lignocaine were used. Through a small supraclavicular incision, the right internal jugular vein was isolated between ligatures and a silastic catheter was passed subcutaneously through the trocar needle to this site from a separate skin puncture on the antero lateral chest wall. Either a 16 gauge (Extracorporeal HC 9305, used earlier in the series) or 18 gauge (Vygon Nutricath ' $\mathrm{S}$ ', used later in the series) silastic catheter was used. Through an internal jugular venotomy the catheter was passed to the level of the upper right atrium (nipple line). Free injection of saline and flow back of blood was ascertained. The vein was ligated in continuity, and wounds were closed with silk sutures and covered with an adherent silastic dressing, which also fixed the catheter. Catheter position was subsequently confirmed on plain chest radiographs. Prophylactic antibiotics were not given. 
All blood products, parenteral nutrition fluids, and antibiotics were infused through the central line, and, occasionally, lines were used for sampling blood. A strict aseptic technique was observed in the preparation of parenteral fluids and in the daily priming and changing of giving sets; otherwise, meticulous handwashing was performed before handling of the line-for example, for the administration of antibiotics. Blood cultures (from peripheral veins if possible) were taken on clinical suspicion of infection. Routine blood cultures were not performed to minimise blood loss. The tips of the central venous catheters were cultured after removal.

\section{Feeding}

The babies received a mixture of $10 \%$ dextrose and electrolyte solution and crystalline amino acid solution with calcium gluconate, mineral and vitamin supplements, and heparin $(100 \mu / 100 \mathrm{ml})$, which was filtered and administered through a constant infusion pump. Intravenous fat emulsion was occasionally used. Milk feeding through nasogastric or nasojejunal tubes was given concurrently whenever tolerated, the aim being to establish complete enteral feeding as soon as possible.

\section{Outcome}

Weight gain. The mean (SD) daily weight gain during the period of use of the central venous line in 36 babies was $17.5(6.3) \mathrm{g} / \mathrm{kg}$. Six babies who died in the first eight days of life are excluded from this analysis.

Complications. Twelve babies died with a central venous line still in situ; six of these succumbed to intraventricular haemorrhage or idiopathic respiratory distress syndrome in the first eight days of life. In the other 30 babies the catheter remained in situ for a mean (SD) of 19.7 (11.9) days (range 3-46 days), and the reasons for its removal are detailed in Table 1. The central line was removed electively from nine babies no longer requiring intravenous alimentation after a mean (SD) of $18.4(7.5)$ days, and blockage of six catheters occurred after a mean (SD) of 20.5 (12.4) days (range 6-44 days). There was no relation between blockage of the line and the flow rate of the infusate. All neck and upper chest swellings resolved upon removal of the central venous line, and all infants in whom there was technical line failure were able to be managed thereafter by the use of peripheral venous infusions.

Eight babies $(19 \%)$ had evidence of systemic infection after the insertion of a central venous
Table 1 Reasons for removal of central venous lines in 42 premature babies

\begin{tabular}{lcc}
\hline & $\begin{array}{l}\text { No (\%) of babies } \\
\text { with this reason }\end{array}$ \\
\hline $\begin{array}{l}\text { Death of baby } \\
\text { Parenteral feeding no longer required } \\
\text { Technical failure: }\end{array}$ & 12 & $(29)$ \\
$\quad \begin{array}{l}\text { Line blocked } \\
\text { Line leaking }\end{array}$ & 9 & $(21)$ \\
$\begin{array}{l}\text { Line accidentally dislodged } \\
\text { Neck and/or upper chest swelling }\end{array}$ & 3 \\
$\begin{array}{l}\text { Positive blood culture(s) } \\
\text { Suspected septicaemia (all cultures subsequently negative) }\end{array}$ & 1 & $(24)$ \\
\hline Total & 5 & $(12)$ \\
\hline
\end{tabular}

catheter (Table 2). In five cases the central venous line was removed because of positive blood culture(s) and the septicaemia was subsequently eradicated, although one baby (case 40) died 13 days after removal of the line from pseudomonas pneumonia. The mean (SD) duration of the use of the central venous catheter in the eight babies who developed systemic infection was 22.3 (11.4) days (range 8-43 days), and in the three who died with the line still in situ was $21.5(10.5)$ days. These last three cases are described in detail.

\section{Case 14}

This girl ( $870 \mathrm{~g}, 26$ weeks' gestation) had a central venous line inserted at eight days for administration of parenteral nutrition after removal of an umbilical arterial catheter. She developed signs of necrotising enterocolitis in the fourth week of life and gradually deteriorated, despite intensive treatment with antibiotics. Blood culture on day 29 grew Pseudomonas aeruginosa and, after death from hepatorenal failure three days later, all bacteriological specimens taken at postmortem examination, including the tip of the central venous line, grew Pseudomonas aeruginosa, Klebsiella pneumoniae, and Proteus mirabilis. There was some friable clot around the tip of the central venous catheter in the superior vena cava, and histological evidence of septic pulmonary emboli.

\section{Case 19}

This girl (885 g, 25 weeks' gestation) had a central venous line inserted on day 2 for administration of intravenous alimentation. She made slow progress and was weaned from intermittent positive pressure ventilation to continuous positive airways pressure on day 27. After sudden collapse and death on day 28 autopsy showed a granulomatous reaction around the tip of the central venous catheter in the superior venal cava, histological evidence of septic emboli in the lungs, and Pityrosporum ovale infection. 


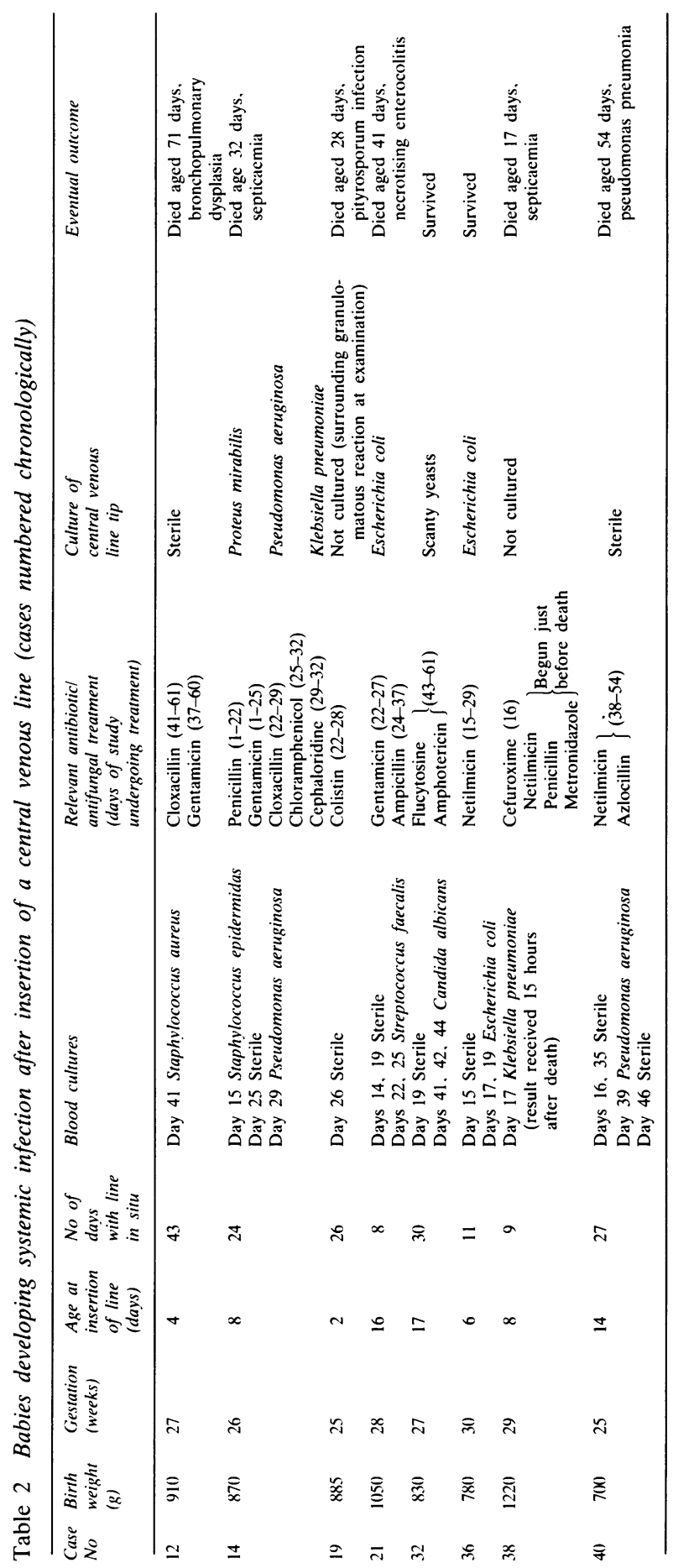


Case 38

This boy (1220 g, 29 weeks' gestation) had a central venous line inserted on day 8 because of failed peripheral venous access. He was successfully weaned off a ventilator and was making good progress until day 17 when he suddenly collapsed and died within a few hours. A blood culture taken shortly before death subsequently grew Klebsiella pneumoniae, and autopsy showed disseminated intravascular coagulation and a massive subarachnoid haemorrhage. There was no evidence of thrombus around the catheter or of embolisation, but the tip of the catheter was not cultured.

In total, four of the 12 babies who died with a central venous line in situ succumbed to septicaemia; in one case (not included in Table 2) the line was inserted after the diagnosis of septicaemia had been confirmed because of impossible peripheral venous access. The causes of death in the other eight infants were intraventricular haemorrhage (five), pneumonia with pneumothorax (one), necrotising enterocolitis (one), and idiopathic respiratory distress syndrome (one). Nine of the 12 infants underwent autopsy, and in all cases the catheter tip was sited in either the superior vena cava or high right atrium, confirming antemortem $x$ ray findings. There was associated loose thrombus in two cases and organised thrombus in one case, but only cases 14 and 19 (vide supra) showed evidence of embolisation.

Six of the eight central venous catheter tips removed from infants with systemic infection were cultured (Table 2). Three yielded the same organism as the blood culture, one grew a different organism, and two were sterile. Of the 25 central venous lines removed from living babies for reasons other than a positive blood culture, four were accidentally contaminated and therefore not sent for culture, 18 were sterile, two grew Staphylococcus epidermidis, and one grew scanty yeasts. The babies from whom the latter three catheters had been withdrawn remained well without treatment.

The only other complication of the use of a central venous line was the development of hydrocephalus in one child, diagnosed at 5 months. It was considered to be due to superior vena caval thrombosis because of associated plethora and venous congestion of the face and upper chest. ${ }^{2}$ The line had been removed after 17 days on suspicion of septicaemia (all cultures were subsequently negative), and superior vena caval thrombosis was not suspected in the neonatal period.

\section{Discussion}

With the increasing survival of extremely preterm infants 'requiring prolonged intensive care, venous access not uncommonly becomes a major problem in a busy neonatal unit. Reliable prolonged venous access through indwelling central venous lines allows minimal handling of sick infants, obviates the problem of tissue necrosis associated with extravasation of hypertonic fluid from tissued peripheral drip sites, ${ }^{3}$ and secures intravenous nutrition, but advantages must be weighed against the risks of sepsis and thrombosis. ${ }^{+8}$

Reported infection rates associated with indwelling central venous lines vary from $7 \%$ to greater than $50 \%{ }^{6-9}$ and depend to a large extent upon the author's definition of 'catheter related sepsis'.

We have attempted to identify all cases of systemic infection occurring after the insertion of a central venous line, thus certainly including some coincidental infections, as it is impossible to assess the role of a central venous line in the development of septicaemia in this very high risk population. Nevertheless, our infection rate of $19 \%$ compares favourably with that of other series concerned mainly with neonates, ${ }^{67}$ particularly as the central venous lines were used for the administration of all blood products and antibiotics, as well as parenteral fluids, and occasionally for blood sampling. Parenteral nutritional fluids must be prepared and administered under strictly aseptic procedures as, in a warm nursery, the infusates provide an excellent culture medium, particularly for candida species, ${ }^{7}$ and violation of asepsis in handling central venous lines greatly increases the risk of infection. ${ }^{8} 10$

To minimise handling of the infant and the risk of disrupting the central venous line the entrance site of the cannula was covered with a sterile transparent occlusive dressing and left undisturbed rather than cleaned and redressed routinely. Regular skin cultures, however, might be a useful indication of colonisation of the cannula. ${ }^{11}$

Regular blood cultures would possibly have detected more cases of septicaemia, thus justifying a greater iatrogenic blood loss. Maintaining a high index of clinical suspicion of infection and removing catheters in cases of confirmed septicaemia was, however, associated with good success in eradication of symptomatic systemic infection. Three infants succumbed to systemic infection with a central venous line still in situ, of whom one (case 14) had a sterile blood culture shortly after first becoming unwell and was terminally ill by receipt of a positive blood culture. The second (case 19) had a sterile blood culture two days before death from fungaemia. The third (case 38) collapsed and died within 24 hours of first becoming unwell from Klebsiella septicaemia. The same organism was grown from a routine throat swab five days before 
death and, in retrospect, a routine blood culture in this case may have detected an early septicaemia. Removal of the central venous line in a collapsed infant, with or without proof of septicaemia, is unlikely to contribute to survival.

Venous thrombosis may result from the use of central venous lines to infuse hypertonic fluids and is known to correlate with long term use of catheter and, inversely, with age in infants receiving central venous alimentation. ${ }^{+}$Organised antemortem thrombus was found around the catheter in the superior vena cava in only one of nine infants undergoing autopsy with a central venous line still in situ and loose thrombus, probably terminal or after death in two. This is in keeping with other findings, ${ }^{5}{ }^{\circ} 8$ although some surviving babies possibly had small thromboses that escaped clinical detection.

One infant developed hydrocephalus, presumed secondary to superior vena caval thrombosis, and it is unlikely that, with regular follow up, this complication has been missed in any other patient. No cases of atrial or ventricular thrombosis, ${ }^{12}$ chylothorax,${ }^{13}$ or clinically detected pulmonary embolisation ${ }^{5}$ were seen.

A recent study from Chicago, concerning the use of central venous catheters for the administration of total parenteral nutrition in infants with birth weight less than $1001 \mathrm{~g}$ reports a mean daily weight gain of $10.4 \mathrm{~g} / \mathrm{kg} .{ }^{6}$ The mean daily weight gain in our series of $17.5 \mathrm{~g} / \mathrm{kg}$ is strong support for the early introduction of milk feeding to the limit of tolerance, complemented by intravenous nutrition. The eight infants who developed systemic infection in our series had a mean daily weight gain of $15.5 \mathrm{~g} / \mathrm{kg}$ during the use of the central venous line $(17 \cdot 0 \mathrm{~g} / \mathrm{kg}$ excluding the three who died with the line still in situ), and so it is unlikely that the lower rate of sepsis in our study contributed appreciably to the better overall weight gain achieved.

This is a retrospective study that covers a period in which there have been many changes in neonatal intensive care and indeed in our criteria for the insertion of central venous catheters. We now prefer to use peripherally inserted, small diameter silicon long lines for parenteral nutrition whenever possible as they can be more easily set up by the resident medical staff and are associated with a lower incidence of sepsis than the larger diameter, surgi- cally placed catheters considered in this series. ${ }^{9}$ It is not always possible, however, to establish peripheral long lines in very small infants, and we believe that, in selected cases, surgical placement of central venous catheters is a useful and potentially life saving procedure in the neonatal intensive care unit.

The authors acknowledge the painstaking care of the nursing staff in the Special Care Baby Unit at Glasgow Royal Maternity Hospital. We also thank Miss June Lawson for typing the manuscript.

We thank Dr John MacLaurin and Dr Paul Galea, consultant neonatologists, for their advice and help and for allowing us to study their patients.

\section{References}

1 Dubowitz LMS. Dubowitz V. Goldberg C. Clinical assessment of gestational age in the newborn infant. $J$ Pediatr 1970;77:1-10.

2 Harr FL. Miller CA. Hydrocephalus resulting from superior vena caval thrombosis in an infant. J Neurosurg 1975;42: 597-601.

3 Coran AG, Weintraub WH. Peripheral intravenous nutritional without fat in neonatal surgery. J Pediatr Surg 1977;12:195-9.

${ }^{+}$Fonkalsrud EW, Ament ME, Berquist WE. Burke M. Occlusion of the vena cava in infants receiving central venous hyperalimentation. Surg Gynecol Obstet 1982:154:189-92.

5 Mollitt DL. Golladay ES. Complications of TPN catheterinduced vena caval thrombosis in children less than one year of age. J Pediatr Surg 1983:18:462-7.

- Ogata ES, Schulman S, Raffensperger J, Luck S, Rusnak M. Caval catheterisation in the intensive care nursery: a useful means for providing parenteral nutrition to the extremely low birth weight infant. J Pediatr Surg 1984:19:258-64.

7 Boeckman CR, Krill CE. Bacterial and fungal infections complicating parenteral alimentation in infants and children $J$ Pediatr Surg 1970:5:117-64.

* Ryan JA, Abel RM, Abbott WM, et al. Catheter complications in total parenteral nutrition. $N$ Engl J Med 1974:290:757-61.

${ }^{9}$ Loeff DS, Matlak ME, Black RE, Overall JC, Dolcourt JL, Johnson DG. Insertion of a small central venous catheter in neonates and young infants. J Pediatr Surg 1982:17:944-9.

10 Syndman DR, Murray SA, Kornfield SJ, Majka JA, Ellis CA. Total parenteral nutrition-related infections. $\mathrm{Am} \mathrm{J} \mathrm{Med}$ 1982;73:695-9

"Syndman DR, Gorbea HF. Pober BR. Majka JA. Murray SA. Perry LK. Predictive value of surveillance skin cultures in totalparenteral-nutrition-related infections. Lancet 1982;ii:1385-8.

12 Aaronson IA. Complication of central venous catheter used for parenteral nutrition (letter). J Pediatr Surg. 1983;18:657.

1.3 Curci M. Dibbins A. Bilateral chylothorax in a newborn. J Pediatr Surg 1980;15:663-9.

Correspondence and requests for reprints to Dr P A M Raine, Department of Pacdiatric Surgery. Royal Hospital for Sick Children. Yorkhill, Glasgow G3 8SJ, Scotland.

Received 13 January 1986. 